\title{
Rapid direct identification of positive paediatric blood cultures by MALDI-TOF MS technology and its clinical impact in the paediatric hospital setting
}

Waniganeththi Arachchige Manori Piyumal Samaranayake ${ }^{1 *}$, Suzanne Dempsey ${ }^{1}$, Annaleise R. Howard-Jones ${ }^{1}$, Alexander Conrad Outhred ${ }^{1,2}$ and Alison Margaret Kesson ${ }^{1,2,3}$

\begin{abstract}
Objective: Rapid diagnostic tools are imperative for timely clinical decision making, particularly in bacteraemic patients. This study evaluated the performance of a fast, inexpensive novel in house method for processing positive blood cultures for immediate identification of microorganisms by matrix-assisted laser desorption ionization-time of flight mass spectrometry (Vitek MS bioMérieux). We prospectively analyzed the clinical impact of such method on the management of pediatric patients.

Result: In total, 360 positive blood cultures were included. Among 318 mono-microbial cultures, in-house method achieved correct identification in 270 (85\%) cultures to the species level, whilst 43 (13.5\%) gave no identification, and 7 (2.2\%) gave discordant identifications. Identification of Gram-negative organisms was accurate to both species and genus level in $99 \%$ of isolates, and for Gram positives accuracy was $84 \%$ to genus and $81 \%$ to species level overall, with accuracy of 100\% for Staphylococcus aureus and Enterococcus to the species level. Assessment of the potential impact of direct identification in sixty sequential cases revealed a clear clinical benefit in 35.5\% of cases. Benefits included timely antibiotic rationalization, change of medical intervention, and early confirmation of contamination. This study demonstrates a highly accurate in-house method with considerable potential clinical benefits for paediatric care.
\end{abstract}

Keywords: Direct identification of blood culture, MALDI-TOF mass spectrometry, Clinical impact, Pediatric, Bloodstream infection

\section{Introduction}

Sepsis is associated with high mortality [1]; hence rapid identification of bloodstream pathogens and timely clinical decision making are paramount. Matrix-assisted laser desorption ionization-time of flight mass spectrometry (MALDI-TOF MS) has revolutionized microbiology

\footnotetext{
*Correspondence: thusithaat@yahoo.com

${ }^{1}$ Department of Infectious Diseases and Microbiology, The Children's

Hospital at Westmead, Sydney, Australia

Full list of author information is available at the end of the article
}

laboratories with improved workflow for pathogen identification both from solid media and positive blood culture broths [2-4]. Conventional processing requires overnight incubation of the blood culture broth on solid media prior to MALDI-TOF MS analysis, delaying organism identification by $18-24 \mathrm{~h}$.

Recently, several in-house protocols for direct organism identification from positive blood culture broths have been developed [2,3]. However, these protocols entail either complicated preprocessing methods or short periods of subculture to increase their accuracy [2-8]. 
As a result, these methods can be tedious and expensive [3], and remain controversial [2]. Performance rates vary, such that there is no consensus on best-practice [2, 9-11]; commercial kits are neither cost-effective nor superior in performance compared to in-house methods [12]. Additionally, most studies to date have focused on adult populations and on the specific Bruker Daltonics Biotyper system [3].

Direct MALDI-TOF MS identification greatly reduces time to pathogen identification $[4,5]$. In a single center open-label controlled clinical trial, Osthoff et al. demonstrated significant reductions in time to pathogen identification and admissions to the intensive care unit, but no difference in mortality, length of stay in a rapid identification group compared to routine methods [5]. Cost-savings for the laboratory from direct MALDI-TOF identification have also been documented [2-4]. On this basis, we developed a simple fast economical in-house method for accurate identification of positive pediatric blood culture broths using the Vitek MS (bioMérieux) system. Subsequently, we prospectively evaluated the clinical outcome of such a method on pediatric inpatient care.

\section{Main text \\ Methods: Study setting}

This study was conducted at the bacteriology laboratory at the Children's Hospital at Westmead, Australia from November 2018 to November 2019. All positive blood culture broths were processed routinely alongside the direct identification protocol for performance comparison.

\section{Routine method}

Blood was taken with aseptic technique, directly inoculated into aerobic (BacT/Alert FA plus), and/or anaerobic bottles (BacT/Alert FN plus). Bottles were loaded onto the BACT/ALERT ${ }^{\circledR}$ VIRTUO $^{\circledR}$ instrument (bioMérieux) for incubation up to 5 days, or until they signaled as positive. All positive blood cultures were analyzed by Gram stain and subjected to subculture on solid media (blood, MacConkey, chocolate and anaerobic blood agar) then incubated at $35 \pm 1{ }^{\circ} \mathrm{C}$ in a $5 \% \mathrm{CO}_{2}$ atmosphere for 18-24 h. Conventional identification methods included biochemical and automated platforms (Vitek II, bioMérieux and Vitek MS, bioMérieux), depending on the organism isolated. Discordant identifications were further characterized by $16 \mathrm{~S}$ rRNA gene sequencing at a reference laboratory when identification was deemed to be clinically relevant.

\section{In-house rapid identification protocol}

For each positive culture, $1.5 \mathrm{ml}$ of positive blood culture broth was centrifuged at $500 \mathrm{rpm}(23 g)$ for $2 \mathrm{~min}$ to separate erythrocytes from the bacterial cells. $50 \mu \mathrm{l}$ of Triton $(10 \times)$ was added to the supernatant followed by gentle mixing and centrifugation for $1 \mathrm{~min}$ at 13,000 rpm $(15,900 \mathrm{~g})$. The pellets were re-suspended in $1.5 \mathrm{ml}$ distilled water and centrifuged for $1 \mathrm{~min}$ at 13,000 rpm $(15,900 g) .50 \mu \mathrm{l}$ of formic acid $(70 \%)$ and $50 \mu \mathrm{l}$ of acetonitrile were added to the pellet and mixed, then centrifuged for $1 \mathrm{~min}$ at 13,000 rpm $(15,900 \mathrm{~g})$. Following this, $1 \mu \mathrm{l}$ of supernatant was spotted in triplicate onto a target slide and air-dried. Each spot was covered with $1 \mu \mathrm{l}$ of an alpha-cyano-4-hydroxycinnamic acid matrix for MALDITOF MS analysis.

\section{MALDI-TOF MS analysis}

The target plate was analyzed by the Vitek ${ }^{\circledR}$ MS V3.2 bioMérieux system and matched against a library of data representing 1316 taxa. This analysis returned a result with the best identification match along with a confidence percentage from 0 to $99.9 \%$. A 95 to $99.9 \%$ confidence reading on a minimum of one spot was considered high at the species level while $90-94 \%$ confidence was considered high at the genus level. If the confidence score was $\geq 50-94 \%$, the result was recorded as 'genuslevel' if there was a choice of 2-4 organisms all within the same genus. However, if the organisms were distributed between different genera, no valid identification was recorded.

\section{Clinical data collection}

A prospective clinical impact analysis was conducted over 7 weeks between August and September 2019. For all positive blood cultures identified over this period, a clinical impact assessment was performed against three categories: (1) change in antimicrobials, (2) change in intervention or (3) infection control impacts. Clinical data pertaining to these outcomes, as well as clinical diagnosis and likely significance of a positive culture, were recorded on day one (day of Gram stain result) and day two (day of formal organism identification) after collection of the index culture. Cases where rapid MALDITOF gave organism identification were considered as having the potential to change clinical management on day one.

\section{Statistical analysis}

The identification results of the in-house method were compared with that of the routine identification method. The correct identification rate was recorded as the number of isolates that were correctly identified by routine methods divided by the overall number of isolates in sub-groups. Sensitivity, specificity, positive predictive values and negative predictive values to genus level were 
calculated to determine diagnostic accuracy using SPSS version 17.

\section{Results}

A total of 360 positive pediatric blood culture broths were collected over the study period. Of these, 308 were from aerobic and 52 from anaerobic bottles. There were 320 mono-microbial infections, and 40 poly-microbial infections. There were two Gram-positive bacteria that were identified only by $16 \mathrm{~S}$ rRNA gene sequencing and these were excluded from the analysis, Table 1 presents microorganism identification results of the positive mono-microbial blood cultures that were analyzed by the routine method and in-house protocol.

\section{Performance of in house method}

As shown in Table 1, 99\% (106/107) of Gram-negative bacteria were identified by the direct method to species level. Gram-positive organisms were accurately identified at a lower rate of $84 \%(168 / 201)$ to the genus level and $81 \%(164 / 201)$ to species level, but this varied greatly by taxa. Identification to the species level was less accurate for coagulase-negative staphylococci $(82 \%)$ and for Gram-positive rods (65\%). Overall, positive predictive values for the direct identification of both Gram-positive and Gram-negative bacteria from mono-microbial blood culture broths to genus level were $96 \%$ and $100 \%$ respectively. None of the yeast isolates were identified by this method.

Forty blood cultures were confirmed by routine methods to be composed of 2 or 3 bacterial species. Of the 40 poly-microbial blood cultures, direct MALDI-TOF MS correctly reported one of the species present in 23 (58\%) of cultures. Identification rates increased to $34(85 \%)$ when the confidence score was lowered to $50-99.9 \%$. The in-house method identified all organisms from $5 / 40$ (13\%) poly-microbial blood culture bottles, with triplicate analysis enabling increased accuracy in these mixed organism samples.

\section{Clinical outcome}

Of the 60 individual patient cases reviewed, 32 were females and 28 were males. The majority (42) were between 1 month to 6 years old. Direct identification yielded a genus or species in $88 \%(53 / 60)$ of cultures. Six specimens did not yield organism identification, and one was a discordant result. Twenty-two specimens represented repeat samples from the same patient with the same organism. Therefore, the clinical impact was determined for the remaining 31 patients' cases. In eleven cases $(35.5 \%)$ early MALDI-TOF identification would have had clinical benefit (Table 2). In ten cases (32.3\%) a change of antimicrobials would have been facilitated, six cases (19.4\%) could have received medical and surgical interventions. No cases had the potential for an infection control intervention.

\section{Clinical case}

A 9 day old term neonate presented in septic shock due to methicillin-susceptible Staphylococcus aureus urosepsis in the context of severe right-sided hydronephrosis with unilateral pelvic-ureteric and vesico-ureteric junction obstruction (Fig. 1). After a 3 days period on extracorporeal membrane oxygenation, nephrostomy insertion and clinical stabilization, he was completing a 4 week course of IV cephazolin when deteriorated with new fevers and haemodynamic instability. Blood and urine cultures were drawn and empiric vancomycin and gentamicin commenced alongside cephazolin.Rapid identification of positive blood culture broth by our in-house method revealed Stenotrophomonas maltophilia, subsequently confirmed on the blood and urine cultures; cerebrospinal fluid cultures were negative. Early identification facilitated timely switch to an appropriate antibiotic regime of single agent intravenous (IV) trimethoprim/sulfamethoxazole (cotrimoxazole) and removal of nephrostomy tube. The child was discharged following a 10 days course of IV cotrimoxazole with full recovery.

\section{Discussion}

This study demonstrates for the first time the utility of rapid identification of positive blood cultures in a pediatric inpatient setting and the impact of this diagnostic approach on clinical decision making.

In this study, we modified a previous protocol [13] to optimize bacterial purity and ease of analysis, including pre-separation of the bacterial pellet from the erythrocytes. The technique was inexpensive, and identification results could be obtained within $15 \mathrm{~min}$ of the blood culture flagging positive. Results of the in-house method were highly consistent with single colony identification for Gram-negative organisms to species and genus level (up to 99\%), outperforming comparable methods in the literature which reported $<90 \%$ concordance $[3,12-16]$.

Whilst identification had high accuracy for Gram negative bacteria as well as S. aureus and Enterococcus spp., the method performed less well for other Gram positive organisms and yeasts, consistent with other direct MALDI-TOF MS methodologies [3, 1416]. The thicker cell walls and spore-forming nature of bacilli $[8,12-16]$ and the more complex processing required to disrupt yeast cell walls [19] are likely contributors. Some studies, using more complicated and costly extraction procedures, have reported slightly higher success rates for Gram positive organisms [17, 18]. Three out of 14 viridans group Streptococci were 
Table 1 List of the microorganisms identified by routine method and direct in house method

\begin{tabular}{|c|c|c|c|c|c|c|c|c|}
\hline $\begin{array}{l}\text { List of microorganisms } \\
\text { identified by routine } \\
\text { methods/number } \\
\text { of isolates }\end{array}$ & $\begin{array}{l}\text { Number } \\
\text { of isolates } \\
\text { identified } \\
\text { by MALDI-TOF } \\
\text { MS at the species } \\
\text { level }\end{array}$ & $\begin{array}{l}\text { Number } \\
\text { of isolates } \\
\text { identified } \\
\text { by MALDI-TOF } \\
\text { MS at the genus } \\
\text { level only }\end{array}$ & $\begin{array}{l}\text { Isolates } \\
\text { not identified } \\
\text { by MALDI-TOF } \\
\text { MS }\end{array}$ & $\begin{array}{l}\text { Isolates } \\
\text { with discordant } \\
\text { results }\end{array}$ & $\begin{array}{l}\text { Sensitivity } \\
(\%)\end{array}$ & $\begin{array}{l}\text { Specificity } \\
\text { (\%) }\end{array}$ & $\begin{array}{l}\text { PPV } \\
\text { (\%) }\end{array}$ & $\begin{array}{l}\text { NPV } \\
(\%)\end{array}$ \\
\hline Staphylococcus aureus (36) & 36 & - & - & & 100 & 100 & 100 & 100 \\
\hline $\begin{array}{l}\text { Staphylococcus epidermidis } \\
\text { (46) }\end{array}$ & 45 & 1 & - & & 82.1 & 97.9 & 93.3 & 94.2 \\
\hline Staphylococcus hominis (9) & 8 & - & 1 & & & & & \\
\hline $\begin{array}{l}\text { Staphylococcus haemolyti- } \\
\text { cus (2) }\end{array}$ & 2 & - & - & & & & & \\
\hline Staphylococcus capitis (3) & 2 & - & 1 & & & & & \\
\hline Staphylococcus cohnii (1) & - & - & 1 & & & & & \\
\hline Staphylococcus simulans (2) & 2 & - & - & & & & & \\
\hline Kocuria kristinae (1) & 1 & - & - & & & & & \\
\hline Dolosigranulum pigrum (1) & - & - & 1 & & & & & \\
\hline Rothia mucilaginosa (2) & 2 & - & - & & & & & \\
\hline Micrococcus luteus (16) & 7 & - & 9 & $\begin{array}{l}\text { L. monocytogenes } \\
(2) \\
\text { L. adecarboxylata } \\
\text { (2) } \\
\text { P. baroniae (1) }\end{array}$ & & & & \\
\hline Micrococcus terreus (1) & - & - & 1 & & & & & \\
\hline $\begin{array}{l}\text { Total GPC resembling } \\
\text { Staphylococcus (120) }\end{array}$ & 105 & 1 & 14 & & 88.3 & 97.5 & 95.5 & 93.2 \\
\hline Streptococcus agalactiae (7) & 6 & - & 1 & & & & & \\
\hline $\begin{array}{l}\text { Streptococcus pneumoniae } \\
\text { (6) }\end{array}$ & 5 & - & 1 & & & & & \\
\hline $\begin{array}{l}\text { Streptococcus mitis/oralis } \\
\text { group (9) }\end{array}$ & 5 & - & 4 & & & & & \\
\hline $\begin{array}{l}\text { Streptococcus parasanguinis } \\
\text { (2) }\end{array}$ & 2 & - & - & & & & & \\
\hline Streptococcus sanguinis (1) & 1 & - & - & & & & & \\
\hline Streptococcus intermedius (1) & 1 & - & - & & & & & \\
\hline $\begin{array}{l}\text { Other Viridans group of } \\
\text { Streptococci (6) }\end{array}$ & - & 2 & 4 & & & & & \\
\hline Streptococcus constellatus (1) & - & - & 1 & & & & & \\
\hline Granulicatella adiacens (1) & 1 & - & - & & & & & \\
\hline Abiotrophia defective (2) & 1 & - & - & $\begin{array}{l}\text { L. adecarboxylata } \\
\text { (1) }\end{array}$ & & & & \\
\hline Enterococcus faecalis (23) & 23 & - & - & & 100 & 100 & 100 & 100 \\
\hline Enterococcus faecium (2) & 2 & - & - & & & & & \\
\hline $\begin{array}{l}\text { Total GPC resembling Strep- } \\
\text { tococcus (61) }\end{array}$ & 47 & 2 & 12 & & 80.3 & 99.6 & 98 & 95.5 \\
\hline Clostridium perfringen (2) & 2 & - & - & & & & & \\
\hline Clostridium septicum (1) & 1 & - & - & & & & & \\
\hline Bacillus cereus (5) & 4 & - & 1 & & & & & \\
\hline Bacillus megaterium (1) & 1 & - & - & & & & & \\
\hline $\begin{array}{l}\text { Bacillus altitudinis/pumilus } \\
\text { (1) }\end{array}$ & 1 & - & - & & & & & \\
\hline Brevibacterium luteolum (1) & 1 & - & - & & & & & \\
\hline Bacillus simplex (1) & 1 & - & - & & & & & \\
\hline Bacillus gibsonii (1) & 1 & - & - & & & & & \\
\hline Dermabacter hominis (1) & - & - & 1 & & & & & \\
\hline
\end{tabular}


Table 1 (continued)

\begin{tabular}{|c|c|c|c|c|c|c|c|c|}
\hline $\begin{array}{l}\text { List of microorganisms } \\
\text { identified by routine } \\
\text { methods/number } \\
\text { of isolates }\end{array}$ & $\begin{array}{l}\text { Number } \\
\text { of isolates } \\
\text { identified } \\
\text { by MALDI-TOF } \\
\text { MS at the species } \\
\text { level }\end{array}$ & $\begin{array}{l}\text { Number } \\
\text { of isolates } \\
\text { identified } \\
\text { by MALDI-TOF } \\
\text { MS at the genus } \\
\text { level only }\end{array}$ & $\begin{array}{l}\text { Isolates } \\
\text { not identified } \\
\text { by MALDI-TOF } \\
\text { MS }\end{array}$ & $\begin{array}{l}\text { Isolates } \\
\text { with discordant } \\
\text { results }\end{array}$ & $\begin{array}{l}\text { Sensitivity } \\
(\%)\end{array}$ & $\begin{array}{l}\text { Specificity } \\
\text { (\%) }\end{array}$ & $\begin{array}{l}\text { PPV } \\
\text { (\%) }\end{array}$ & $\begin{array}{l}\text { NPV } \\
(\%)\end{array}$ \\
\hline Actinomyces viscosus (1) & - & - & 1 & & & & & \\
\hline $\begin{array}{l}\text { Curtobacterium flaccumfa- } \\
\text { ciens (1) }\end{array}$ & - & - & 1 & & & & & \\
\hline Dietzia cinnamea (1) & - & - & 1 & S. cornosus (1) & & & & \\
\hline $\begin{array}{l}\text { Corynebacterium aurimuco- } \\
\text { sum (1) }\end{array}$ & - & 1 & - & & & & & \\
\hline Corynebacterium spp. (2) & - & - & 2 & & & & & \\
\hline Total Gram positive rods (20) & 12 & 1 & 7 & & 65 & 99.7 & 92.9 & 97.7 \\
\hline Escherichia coli (29) & 29 & - & - & & & & & \\
\hline Klebsiella pneumoniae (11) & 11 & - & - & & & & & \\
\hline Klebsiella oxytoca (2) & 2 & - & - & & & & & \\
\hline $\begin{array}{l}\text { Enterobacter cloacae com- } \\
\text { plex (22) }\end{array}$ & 21 & - & 1 & & & & & \\
\hline $\begin{array}{l}\text { Salmonella enterica subsp. } \\
\text { enterica (14) }\end{array}$ & 14 & - & - & & & & & \\
\hline Serratia marcescens (4) & 4 & - & - & & & & & \\
\hline Morganella morganii (1) & 1 & - & - & & & & & \\
\hline Pseudomonas aeruginosa (9) & 9 & - & - & & & & & \\
\hline $\begin{array}{l}\text { Acinetobacter radioresistans } \\
\text { (1) }\end{array}$ & 1 & - & - & & & & & \\
\hline Acinetobacter /woffii (1) & 1 & - & - & & & & & \\
\hline Acinetobacter ursingii (1) & 1 & - & - & & & & & \\
\hline Acinetobacter baumannii (1) & 1 & - & - & & & & & \\
\hline Acinetobacter johnsonii (1) & 1 & - & - & & & & & \\
\hline $\begin{array}{l}\text { Stenotrophomonas malt- } \\
\text { ophilia (7) }\end{array}$ & 7 & - & - & & & & & \\
\hline Neisseria meningitides (1) & 1 & - & - & & & & & \\
\hline Moraxella catarrhalis (2) & 2 & - & - & & & & & \\
\hline Total Gram negatives (107) & 106 & - & 1 & & 99 & 100 & 100 & 99.5 \\
\hline Candida albicans (1) & - & - & 1 & & & & & \\
\hline Candida parapsilosis (9) & - & - & 9 & & & & & \\
\hline Total yeast (10) & - & - & 10 & & & & & \\
\hline
\end{tabular}

MALDI-TOF matrix assisted laser desorption ionization-time of flight, $P P V$ positive predictive value, NPV negative predictive value

misidentified as S. pneumoniae due to the inherent difficulty of MALDI-TOF MS in identifying closely related species. This problem has also been documented with the commercial Bruker method [8]. Nevertheless, positive predictive value for $S$. pneumoniae stayed at a higher level (100\%) in our assay. Our study was underpowered to adequately assess accuracy for Gram positive anaerobes (e.g. Clostridium spp.). Thus, the direct method should be interpreted carefully for potential coagulase-negative staphylococci and Gram-positive bacilli until there is formal identification. Our assay out-performed most published studies $[3,12]$; detecting at least one species from $85 \%$ of poly-microbial blood cultures with a lowered confidence score. Low organism counts or competition from impurities may explain the lower confidence scores in poly-microbial samples.

There have been very few studies focusing on the impact of rapid identification from MALDI-TOF MS for both the patient and the laboratory [4, 5, 20-24]. Results from our study demonstrate that direct identification of organisms in positive blood culture bottles using MALDI-TOF MS could have important clinical impacts. The potential impact was most significant for the more virulent organisms (S. aureus, Streptococcus 
Table 2 Summary of cases for which rapid organism identification would have impacted clinical decision making

\begin{tabular}{|c|c|c|c|c|c|c|c|}
\hline Case number & $\begin{array}{l}\text { Gram stain day } \\
\text { one }\end{array}$ & Clinical context & $\begin{array}{l}\text { Antibiotics } \\
\text { on day one }\end{array}$ & $\begin{array}{l}\text { Organisms } \\
\text { identified } \\
\text { by direct } \\
\text { MALDI-ToF } \\
\text { on day one }\end{array}$ & $\begin{array}{l}\text { Organisms } \\
\text { identified } \\
\text { from sub- } \\
\text { culture day two }\end{array}$ & $\begin{array}{l}\text { Antibiotic } \\
\text { change on day } \\
\text { two }\end{array}$ & $\begin{array}{l}\text { Medical/surgical } \\
\text { intervention }\end{array}$ \\
\hline 1 & GNR & Pyelonephritis & $\begin{array}{l}\text { Vancomycin and } \\
\text { Gentamicin }\end{array}$ & S. maltophilia & S. maltophilia & $\begin{array}{l}\text { Cefotaxime and } \\
\text { Cotrimoxazole } \\
\text { added }\end{array}$ & $\begin{array}{l}\text { Neprostomy tube } \\
\text { removed }\end{array}$ \\
\hline 2 & GNR & $\begin{array}{l}\text { Febrile neutrope- } \\
\text { nia due to UTI }\end{array}$ & $\begin{array}{l}\text { Piperacillin/Tazo- } \\
\text { bactam and } \\
\text { Gentamicin }\end{array}$ & E. coli & E. coli & $\begin{array}{l}\text { Cefotaxime } \\
\text { added. } \\
\text { Gentamicin } \\
\text { continued }\end{array}$ & - \\
\hline 3 & GNR & $\begin{array}{l}\text { Post-operative } \\
\text { liver transplant } \\
\text { infection }\end{array}$ & No antibiotics & $\begin{array}{l}\text { Enterobacter } \\
\text { cloacae com- } \\
\text { plex }\end{array}$ & $\begin{array}{l}\text { Enterobacter } \\
\text { cloacae com- } \\
\text { plex }\end{array}$ & Imipenem added & $\begin{array}{l}\text { Interventions to } \\
\text { sterilise line with } \\
\text { antibiotic locks }\end{array}$ \\
\hline 4 & GPR & Myonecrosis & $\begin{array}{l}\text { Piperacillin/Tazo- } \\
\text { bactam, Clin- } \\
\text { damycin and } \\
\text { Vancomycin }\end{array}$ & C. septicum & C. septicum & $\begin{array}{l}\text { High dose } \\
\text { benzyl penicil- } \\
\text { lin added } \\
\text { clindamycin } \\
\text { continued; } \\
\text { tazocin and } \\
\text { vancomycin } \\
\text { ceased }\end{array}$ & $\begin{array}{l}\text { Urgent re-explo- } \\
\text { ration of leg } \\
\text { as necrotising } \\
\text { fasciitis }\end{array}$ \\
\hline 5 & GPCSTR & $\begin{array}{l}\text { Intra muscular } \\
\text { abscess }\end{array}$ & $\begin{array}{l}\text { Penicillin and } \\
\text { Flucloxacillin }\end{array}$ & S.parasanguinis & S.parasanguinis & $\begin{array}{l}\text { Penicillin contin- } \\
\text { ued Flucloxacil- } \\
\text { lin stopped }\end{array}$ & $\begin{array}{l}\text { MRI to rule out } \\
\text { septic arthritis }\end{array}$ \\
\hline 6 & GPCSTR & Meningitis & $\begin{array}{l}\text { Cefotaxine, Gen- } \\
\text { tamicin and } \\
\text { Ampicillin }\end{array}$ & S. agalactiae & S. agalactiae & $\begin{array}{l}\text { Benzylpenicillin } \\
\text { added } \\
\text { Cefotaxime, } \\
\text { Gentamicin, } \\
\text { Ampicillin } \\
\text { ceased }\end{array}$ & - \\
\hline 7 & GPCSTA & $\begin{array}{l}\text { Congenital heart } \\
\text { disease with } \\
\text { fever }\end{array}$ & $\begin{array}{l}\text { Vancomycin and } \\
\text { Gentamicin }\end{array}$ & S. epidermidis & S. epidermidis & $\begin{array}{l}\text { Vancomycin } \\
\text { continued } \\
\text { Gentamicin } \\
\text { ceased }\end{array}$ & - \\
\hline 8 & GPCSTA & $\begin{array}{l}\text { Febrile neutro- } \\
\text { penia }\end{array}$ & $\begin{array}{l}\text { Piperacillin/Tazo- } \\
\text { bactam and } \\
\text { Vancomycin }\end{array}$ & S. epidermidis & S. epidermidis & $\begin{array}{l}\text { Vancomycin } \\
\text { continued } \\
\text { Piperacillin/Tazo- } \\
\text { bactam ceased }\end{array}$ & CVL removed \\
\hline 9 & GPCSTA & $\begin{array}{l}\text { Primary immune } \\
\text { deficiency with } \\
\text { fever }\end{array}$ & $\begin{array}{l}\text { Piperacillin/Tazo- } \\
\text { bactam and } \\
\text { Vancomycin }\end{array}$ & S. epidermidis & S. epidermidis & $\begin{array}{l}\text { Piperacillin/Tazo- } \\
\text { bactam and } \\
\text { Vancomycin } \\
\text { continued }\end{array}$ & CVL removed \\
\hline 10 & GPCSTA & $\begin{array}{l}\text { Acute bronchi- } \\
\text { olitis }\end{array}$ & Vancomycin & S. epidermidis & S. epidermidis & $\begin{array}{l}\text { Vancomycin } \\
\text { ceased as } \\
\text { probable con- } \\
\text { tamination }\end{array}$ & - \\
\hline 11 & GPCSTR & Cerebral palsy & Vancomycin & S. mitis & S. mitis & $\begin{array}{l}\text { Vancomycin } \\
\text { ceased as } \\
\text { probable con- } \\
\text { tamination }\end{array}$ & - \\
\hline
\end{tabular}

MALDI-TOF matrix assisted laser desorption ionization-time of flight, GNR Gram negative rods, GPR Gram positive rods, GPCSTR Gram positive Staphylococci, GPCSTA Gram positive Streptococci, CVL central venous line, UTI urinary tract infection

spp.) and organisms with potential resistance to cephalosporins (Enterobacter cloacae), and carbapenems (Stenotrophomonas maltophilia) due to required deviation from empiric therapeutic regimens. In patients with central venous access device (CVAD) infections, early identification had clear benefits for prompt CVAD removal. Our results suggested that narrow spectrum therapy could be advised from day one when a common pathogen with predictable susceptibility profile is identified such as Staphylococcus aureus or Enterococcus 


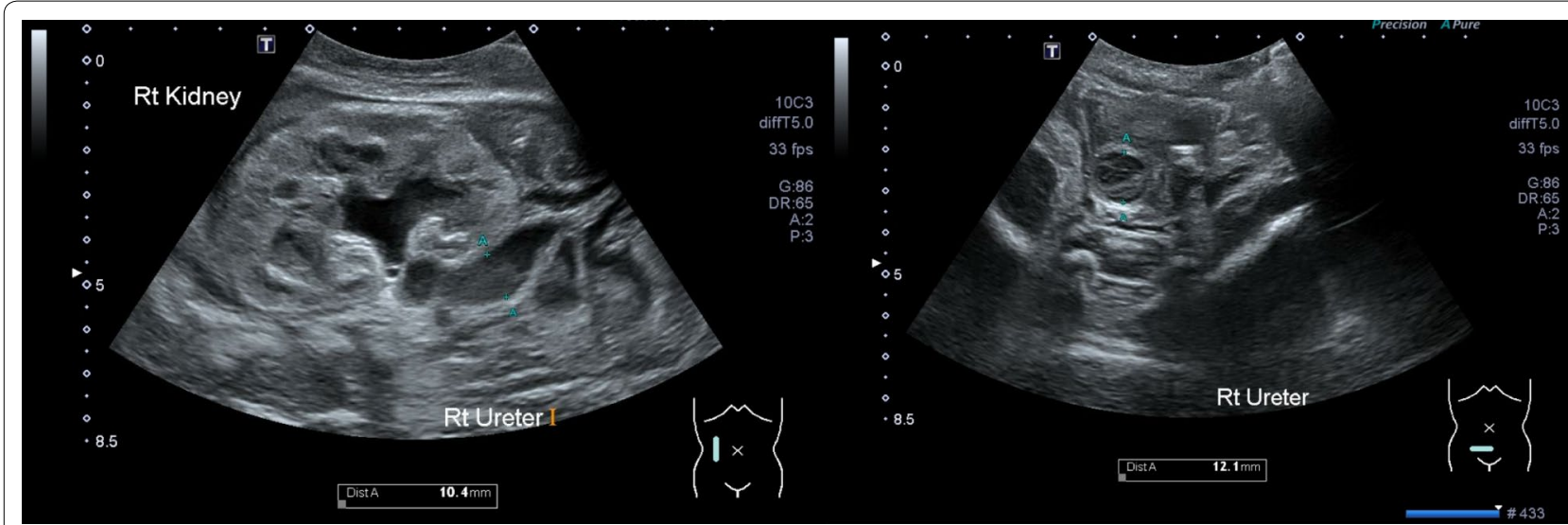

Fig. 1 Renal tract ultrasound showing appearance of hydro-nephrosis of right kidney and dilated right ureter (diameter $12.1 \mathrm{~mm}$ ) on initial presentation. Rt Kidney right kidney, Rt Ureter right ureter, Dist A distance A (12.1 mm)

faecalis. Early confirmation of contaminated blood cultures was advantageous, leading to potential de-escalation of antibiotics along with complimentary diagnostic testing and shortening of hospital stay, in line with previous studies $[2,5,22]$. Although, clinical impact on infection control was not noteworthy, there was a case of $N$. meningitidis bacteremia which helped to early escalation of infection prevention strategies during the first phase of the study.

We have demonstrated that this technique is highly accurate for Gram negative organisms and Staphylococcus aureus, and has potential impacts on length of stay and antibiotic rationalization and infection control decision making. The reliability, rapidity, and simplicity of our technique allow it to be easily adopted by modern microbiology laboratories equipped with MALDI-TOF MS technology.

\section{Limitation}

Our in-house method was incapable of identifying yeast and a randomized controlled study in a larger population would enable assessment of clinical outcomes to a larger degree.

\section{Abbreviations \\ MALDI-TOF-MS: matrix assisted laser desorption ionization-time of flight mass spectrometry; PCR: polymerase chain reaction; 16s rRNA: 16S ribosomal ribonucleic acid; CVL: central venous line; PPV: positive predictive value; GNR: Gram negative rods; GPR: Gram positive rods; GPCSTA: Gram positive cocci resembling Staphylococci; GPCSTR: Gram positive cocci resembling Strepto- cocci; CLABSI: central venous line-associated bloodstream infection.}

\section{Acknowledgements}

We would like to thank the staff of Department of bacteriology for their support with special thanks to Dr. Leonard Moaven for his assistance in literature search and reviewing data during this study.

\section{Authors' contributions}

WAMPS, AMK, ACO: design of the study: WAAMP, SD: acquisition of data. WAMPS, AMK, ACO, AH, SD: troubleshooting, evaluation of data, preparation of the manuscript, and reviewing manuscript. All authors read and approved the final manuscript.

\section{Funding}

Not applicable.

\section{Availability of data and materials}

The datasets used during the current study are available from the corresponding author on reasonable request.

\section{Ethics approval and consent to participate}

All positive blood cultures were taken as a part of routine practice. The material tested was bacterial and not human in origin. Therefore, administrative or ethical permission was not required. Sydney Children's Hospital Network Human Research Ethics Committee.

\section{Consent for publication}

Parental permission was obtained in writing for use of the de-identified case report and accompanying images.

\section{Competing interests}

The authors declare that they have no competing interests.

\section{Author details}

${ }^{1}$ Department of Infectious Diseases and Microbiology, The Children's Hospital at Westmead, Sydney, Australia. ${ }^{2}$ The Marie Bashir Institute of Infectious Diseases and Biosecurity, The University of Sydney, Sydney, Australia. ${ }^{3}$ Discipline of Child and Adolescent Health, The University of Sydney, Sydney, Australia.

Received: 11 November 2019 Accepted: 14 December 2019

Published online: 06 January 2020

\section{References}

1. Lueangarun S, Leelarasamee A. Impact of inappropriate empiric antimicrobial therapy on mortality of septic patients with bacteremia: a retrospective study. Interdiscip Perspect Infect Dis. 2012. https://doi. org/10.1155/2012/765205.

2. Luethy PM, Johnson JK. The use of matrix-assisted laser desorption/ ionization time-of-flight mass spectrometry (MALDI-TOF MS) for the identification of pathogens causing sepsis. J Appl Lab Med. 2019;3(4):675-85.

3. Faron ML, Buchan BW, Ledeboer NA. Matrix-assisted laser desorption ionization-time of flight mass spectrometry for use with positive blood 
cultures: methodology, performance, and optimization. J Clin Microbiol. 2017;55:3328-38.

4. Philippe RSLW, Heather JA, James AK, Kimberly AN, Paulette FP, Jodi $\mathrm{G}$, et al. Identification of blood culture isolates directly from positive blood cultures by use of matrix-assisted laser desorption ionizationtime of flight mass spectrometry and a commercial extraction system: analysis of performance, cost, and turnaround time. J Clin Microbiol. 2012;50(10):3324-8.

5. Osthoff M, Gürtler N, Bassetti S, Balestra G, Marsch M, Pargger H. Impact of MALDI-TOF-MS-based identification directly from positive blood cultures on patient management: a controlled clinical trial. Clin Microbiol Infect. 2017;23:78-85.

6. Fothergill A, Kasinathan V, Hyman J, Walsh J, Drake T, Wang YF. Rapid identification of bacteria and yeasts from positive-blood-culture bottles by using a lysis-filtration method and matrix-assisted laser desorption ionization-time of flight mass spectrum analysis with the SARAMIS Database. J Clin Microbiol. 2013;51(3):805-9.

7. Bhatti MM, Boonlayangoor S, Beavis KG, Tesi V. Rapid identification of positive blood cultures by matrix-assisted laser desorption ionizationtime of flight mass spectrometry using prewarmed agar plates. J Clin Microbiol. 2014;52(12):4334-8.

8. Yonetani S, Ohnishi H, Ohkusu K, Matsumoto T, Watanabe T. Direct identification of microorganisms from positive blood cultures by MALDI-TOF MS using an in-house saponin method. Int J Infect Dis. 2016;52:37-42.

9. Tanner H, Evans JT, Gossain S, Hussain A. Evaluation of three sample preparation methods for the direct identification of bacteria in positive blood cultures by MALDI-TOF. BMC Res Notes. 2017;10:48.

10. Zhou M, Yang Q, Kudinha T, Sun L, Zhang R, Liu C, et al. An improved in-house MALDI-TOF MS protocol for direct cost-effective identification of pathogens from blood cultures. Front Microbiol. 2017;8:1824. https://doi. org/10.3389/fmicb.

11. Lin JF, Ge MC, Liu TP, Chang SC, Lu JJ. A simple method for rapid microbial identification from positive monomicrobial blood culture bottles through matrix-assisted laser desorption ionization time-of-flight mass spectrometry. J Microbiol Immunol Infect. 2018;51:659-65.

12. Chen JH, Ho PL, Kwan GS, She KK, Siu GK, Cheng VC, et al. Direct bacterial identification in positive blood cultures by use of two commercial matrixassisted laser desorption ionization-time of flight mass spectrometry systems. J Clin Microbiol. 2013;51(6):1733-9.

13. Bazzia AM, Rabaanb AA, Edailya ZE, Johna S, Fawaraha MM, Al-Tawfigc JA. Comparison among four proposed direct blood culture microbial identification methods using MALDI-TOF MS. J Infect Public Health. 2017;10:308-15.

14. Rodriguez-Sanchez B, Sanchez-Carrillo C, Ruiz A, Marin M, Cercenado E, Rodriguez-Creixems M, et al. Direct identification of pathogens from positive blood cultures using matrix-assisted laser desorption-ionization time-of-flight mass spectrometry. Clin Microbiol Infect. 2014;20:0421-7.
15. Azrad M, Keness Y, Nitzan O, Pastukh N, Tkhawkho L, Freidus V, et al. Cheap and rapid in-house method for direct identification of positive blood cultures by MALDI-TOF MS technology. BMC Infect Dis. 2019;19:72.

16. La Scola B, Raoult D. Direct Identification of Bacteria in Positive Blood Culture Bottles by matrix-assisted laser desorption ionisation time-of-flight mass spectrometry. PLoS ONE. 2009;4(11):e8041. https://doi.org/10.1371/ journal.pone.0008041.

17. Verroken A, Defourny L, Lechgar L, Magnette A, Delmee M, Glupczynski Y. Reducing time to identification of positive blood cultures with MALDITOF MS analysis after a 5-h subculture. Eur J Clin Microbiol Infect Dis. 2015;34:405-13.

18. Kohlmann R, Hoffmann A, Geis G, Gatermann S. MALDI-TOF mass spectrometry following short incubation on a solid medium is a valuable tool for rapid pathogen identification from positive blood cultures. Int J Med Microbiol. 2015;305:469-79.

19. Yan Y, He Y, Maier T, Quinn C, Shi G, Li H, et al. Improved identification of yeast species directly from positive blood culture media by combining Sepsityper specimen processing and Microflex analysis with the matrix-assisted laser desorption ionization Biotyper system. J Clin Microbiol. 2011;49:2528-32.

20. French $\mathrm{K}$, Evans J, Tanner H, Gossain S, Hussain A. The clinical impact of rapid, direct MALDI-ToF identification of bacteria from positive blood cultures. PLoS ONE. 2016;11(12):e0169332. https://doi.org/10.1371/journ al.pone.0169332.

21. Delport JA, Strikwerda A, Armstrong A, Schaus D, John M. Quality of care is improved by rapid short incubation MALDI-ToF identification from blood cultures as measured by reduced length of stay and patient outcomes as part of a multi-disciplinary approach to bacteremia in pediatric patients. PLOS ONE. 2016;11(8):e0160618. https://doi.org/10.1371/journ al.pone.0160618.

22. Martiny D, Debaugnies F, Gateff D, Gerard M, Aoun M, Martin C, et al. Impact of rapid microbial identification directly from positive blood cultures using matrix-assisted laser desorption/ionization time-of-flight mass spectrometry on patient management. Clin Microbiol Infect. 2013;19(12):568-81.

23. Park JM, Noh JY, Kim MJ, Yun TG, Lee SG, Chung KS, et al. MALDI-TOF mass spectrometry based on parylene-matrix chip for the analysis of lysophosphatidylcholine in sepsis patient sera. Anal Chem. 2019;91(22):14719-27.

24. To KN, Cornwell E, Daniel R, Goonesekera S, Jauneikaite E, Chalker V, et al. Evaluation of matrix-assisted laser desorption ionisation time-of-flight mass spectrometry (MALDI-TOF MS) for the identification of group B Streptococcus. BMC Res Notes. 2019;12(1):85.

\section{Publisher's Note}

Springer Nature remains neutral with regard to jurisdictional claims in published maps and institutional affiliations.
Ready to submit your research? Choose BMC and benefit from:

- fast, convenient online submission

- thorough peer review by experienced researchers in your field

- rapid publication on acceptance

- support for research data, including large and complex data types

- gold Open Access which fosters wider collaboration and increased citations

- maximum visibility for your research: over $100 \mathrm{M}$ website views per year

At BMC, research is always in progress.

Learn more biomedcentral.com/submissions 